\title{
LAS CONSTRUCCIONES AB URBE CONDITA EN GRIEGO, CON ESPECIAL ATENCIÓN A LOS USOS HOMÉRICOS*
}

\section{TOMÁS FERNÁNDEZ}

Universidad Nacional de Buenos Aires - CONICET

tomas.fernandez@conicet.gov.ar

Recibido: 20/03/20

Aceptado: 16/09/20

El presente artículo se propone estudiar las construcciones $a b$ urbe condita en

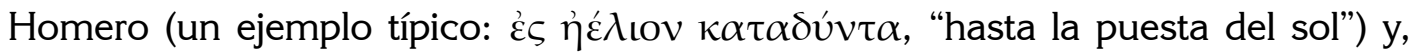
subsidiariamente, en otros autores griegos. Su fin es demostrar que estas construcciones se distinguen de otras análogas por cuatro características, a saber: a) el participio es imprescindible, salvo en un limitado grupo de verbos (de "ser", "llegar a ser" y análogos); b) el participio siempre modifica al núcleo de un término preposicional; c) el participio es predicativo, no atributivo; d) el participio tiene una posición relativamente fija.

Homero / Participios / Ab urbe condita / Gramática

\section{AB URBE CONDITA CONSTRUCTIONS IN GREEK, WITH SPECIAL ATTENTION TO HOMERIC GRAMMAR}

This article will examine $a b$ urbe condita constructions in Homer (a typical example:

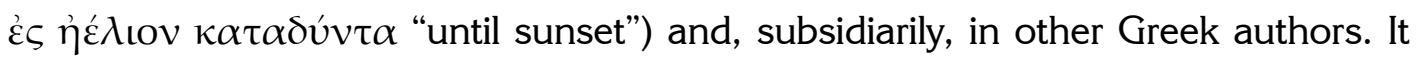
aims to prove that these constructions can be distinguished from other analogous ones by four features, to wit: a) the participle is necessary, except in a limited group of verbs ("to be", "to become" and the like); b) the participle always modifies the nucleus of a prepositional term; c) the participle is predicative, not attributive; d) the participle has a relatively fixed position.

Homer / Participles / Ab urbe condita / Grammar 
1. Entre las muchas construcciones de participio que intrigan desde hace tiempo a los estudiosos de la gramática griega, se destaca una que ha sido definida por analogía con su contraparte latina. Aparece en frases como $\mu \varepsilon \tau \dot{\alpha} \Sigma v \varrho \alpha \kappa o u ́ \sigma \alpha \varsigma$

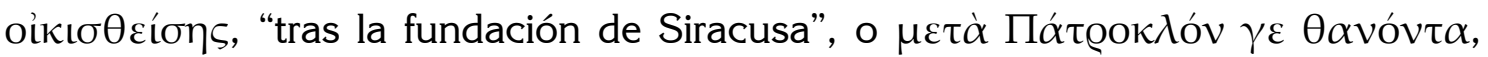
"tras la muerte de Patroclo". La dependencia frente a la conceptualización latina llevó a que se identifique estas construcciones, incluso en griego, como ab urbe condita (de ahora en más AUC), algo que ha motivado, en mi opinión, el descuido de algunos de sus elementos específicos. La definición corriente también coincide con la que proveen las gramáticas latinas: se trata de aquella construcción en la que la mayor carga de significado reside en el participio, no en el sustantivo, motivo por el que se traduce el participio como un sustantivo abstracto ("la fundación") y el sustantivo por una suerte de genitivo ("de la ciudad") ${ }^{1}$. El sustantivo abstracto, que conserva el matiz de acción presente en el participio, indica un evento o suceso (fundación, muerte, etc.).

2. SCHWYZER (1950:404) escribe sobre la construcción AUC: "Seit dem V. Jahrhundert treten die aus den lat. (Nachahmungen) Caesar occisus und ab urbe condita bekannten literarischen Typen auf, die anschaulicher sind als die dafür in neuern Sprachen üblichen Verbalabstrakta mit Genitiv. Sie finden sich außerhalb der Prosa selten"2. La restricción a la prosa seguramente es un resabio de la gramática de STAHL (1907), ya refutado por GILDERSLEEVE (pero seguramente pasado por alto por SCHWYZER 1950) ${ }^{3}$. Más interesante, a los fines de este artículo, es la idea de que no son anteriores al siglo V. Al contrario de lo que supone SCHWYZER (1950), se

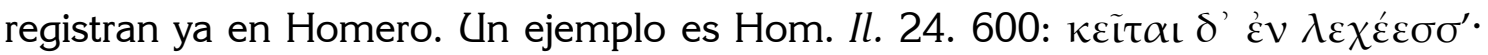

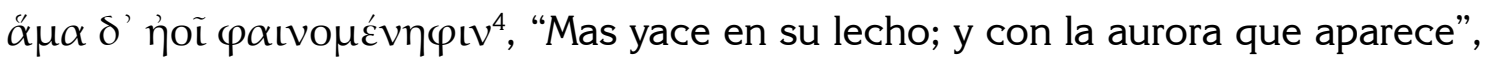
o, diríamos nosotros, "con la aparición de la aurora". Una frase estructuralmente

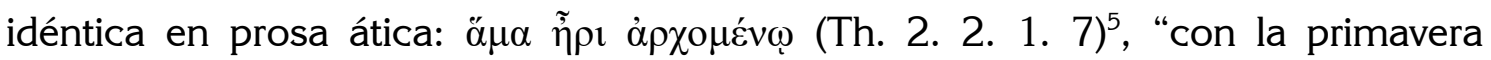
comenzando" o, diríamos, "con el comienzo de la primavera". Un sentido difícilmente distinguible puede expresarse bajo la forma del genitivo absoluto: ع́ $\alpha$ @o

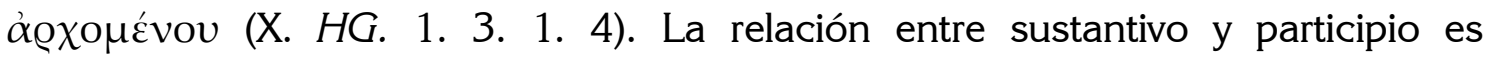
equivalente en ambas construcciones. La construcción AUC, sin embargo, precisa la relación sintáctica entre miembros de la frase; la preposición deja en claro el contenido semántico (o función semántica) expresado por el sintagma6. Así, la indeterminación del genitivo absoluto se ve circunscripta, algo que, en ciertos casos, puede evitar ambigüedades o subrayar un matiz ${ }^{7}$. Más allá de este punto, las diferencias entre una y otra estructura son menores. Con todo, podría arriesgarse la hipótesis de que la construcción AUC haya surgido para precisar el sentido indefinido de ciertas construcciones absolutas.

3. El único estudio sistemático sobre la construcción ab urbe condita en griego fue publicado en 1939 por F.P. JONES. Este erudito asegura que ninguna peculiaridad sintáctica distingue el participio en construcciones AUC de otros participios predicativos: "Now there is nothing in the use of the cases, tenses, voices, or 
negatives to distinguish these participles from others in the same position. The sole criterion is the logical relation found to exist between noun and participle" ${ }^{\text {. Esta }}$ afirmación debería matizarse y eso, precisamente, es lo que pretende este artículo. Es cierto que, en un sentido laxo, podría llamarse construcción AUC a cualquiera en la que la mayor carga semántica residiera en el participio. Esta caracterización, vaga e imprecisa, es de dudosa utilidad. Ciertas construcciones AUC en sentido estricto poseen peculiaridades también sintácticas que permiten diferenciarlas rigurosamente de cualquier otra construcción de participio.

En lo que sigue se contrastarán brevemente las construcciones AUC en sentido estricto y las construcciones AUC en sentido laxo, incluyendo ejemplos no homéricos que ilustrarán los diferentes usos en distintos momentos de la lengua griega.

En este punto, podemos de nuevo citar a SCHWYZER (1950). Según el erudito suizo, las construcciones AUC habrían surgido sobre la base de otras del estilo: ooi

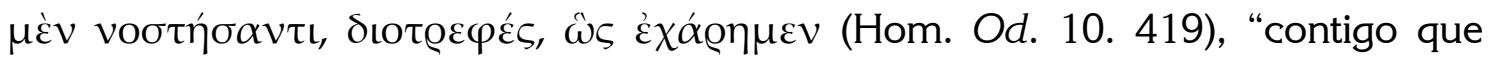
regresabas, retoño de Zeus, cómo nos alegrábamos", reinterpretado como "con tu regreso" ${ }^{2}$. La relación genética entre casos como este y las construcciones AUC en sentido estricto es dudosa; no resulta claro que estas hayan derivado de una construcción de verba affectuum ${ }^{10}$, atestiguada desde antiguo en muchas posiciones, y en la que el participio es exigido antes por el verbo que por el pronombre. La ambigüedad del ejemplo provisto por SCHWYZER prueba hasta qué punto la construcción no entraba de lleno en el debate gramatical.

Otras construcciones AUC en sentido laxo:

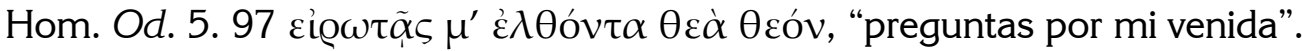

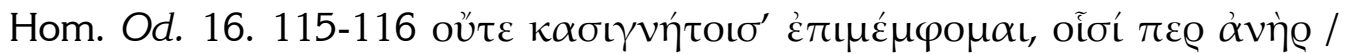

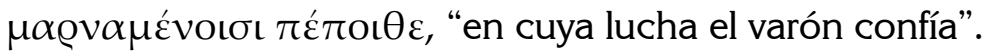

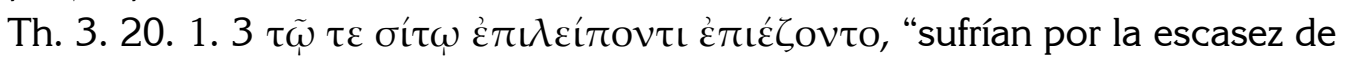
la cosecha" (SмYTH 1956: 456, § 2053) ${ }^{11}$.

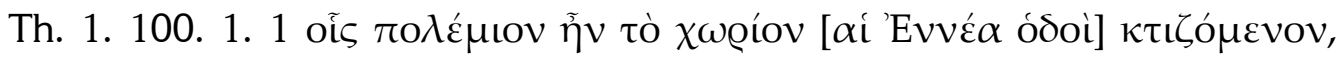
"para quienes el establecimiento de la región era una amenaza" (JONES 1939: 8).

El participio puede eliminarse sin daño, por un lado; por el otro, puede comprenderse formando una paráfrasis con el imperfecto del verbo عỉuí.

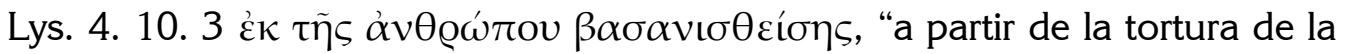
sirvienta" (JONES 1939: 11).

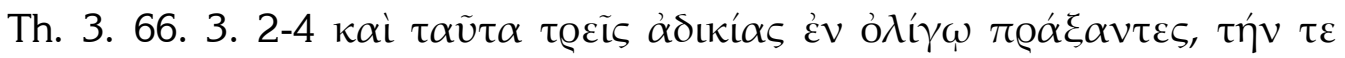

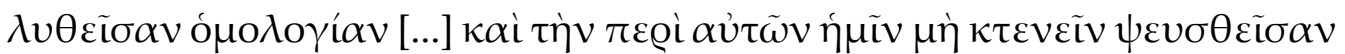
útó $\chi \varepsilon \sigma \iota v$ [...], "la violación de tu acuerdo [...] y la ruptura de tu promesa hacia nosotros" (JONES 1939: 80). 


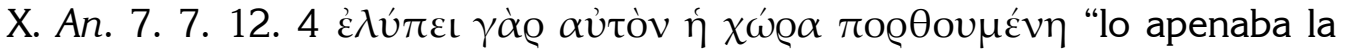
destrucción de la región" (JONES 1939: 17).

Las construcciones AUC en sentido estricto, por su parte, son relativamente escasas:

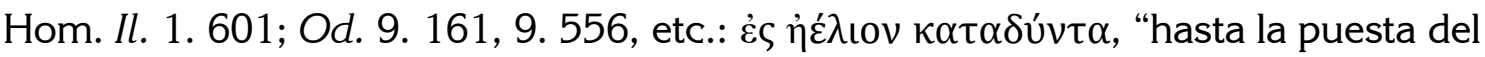
sol". Si el participio faltase, la frase prepositiva sería un simple quo, "hacia el sol".

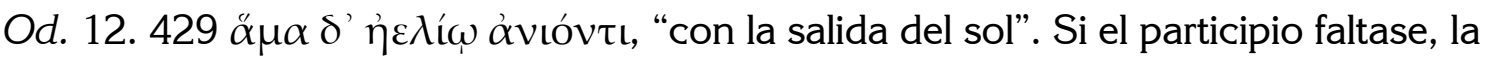

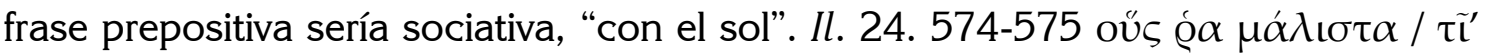

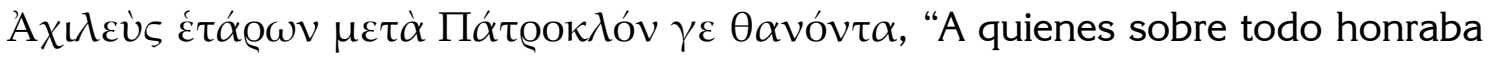
Aquiles tras la muerte de Patroclo" o, más precisamente "después de que Patroclo hubiera muerto", "una vez muerto Patroclo". Si el participio faltase, habría que comprender la frase prepositiva como locativa "en dirección a", no como temporal.

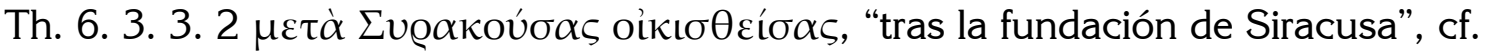
post urbem conditam. Si el participio faltase, habría que comprender la frase prepositiva como sociativa, "con Siracusa", no como temporal ${ }^{12}$.

4. Pueden presentarse unas pocas reglas de reconocimiento, que todavía requieren una elaboración más exhaustiva pero que alcanzan, estimamos, para mostrar que la construcción AUC tiene rasgos funcionales que exceden lo meramente semántico.

a. El participio es imprescindible para la significación, salvo en un limitado grupo de verbos (de "ser", "llegar a ser" y análogos).

b. El participio modifica al núcleo de un término preposicional.

c. El participio es predicativo, no atributivo.

d. El participio tiene una posición relativamente fija, de preferencia después del sustantivo, pero ocasionalmente también antes de la preposición (e. g., Hom. Il. 19.

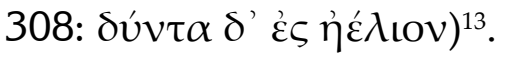

5. Empezamos por lo más fundamental: la construcción AUC se caracteriza por tener un participio exigido. En esto funciona como el participio llamado suplementario o completivo ${ }^{14}$; la diferencia estriba en que en las construcciones AUC el participio modifica al sustantivo, no al verbo principal.

Hemos dicho que, para algunos tipos de verbo como "ser" o "llegar a ser", el participio puede ser eliminado mentalmente: $\alpha \alpha \mu \alpha \delta$ ' joĩ $\varphi \alpha \iota v o \mu \varepsilon ́ v \eta \varphi ı v$ es una construcción AUC en sentido estricto, por más que también ő $\mu$ ' ’̉oĩ (Hom. Od. 16. 2) sea posible. El criterio de la eliminación del participio no parece aplicarse: "con la aurora", simplemente, en vez de "con la aparición de la aurora", no es inaceptable (del mismo modo que "después de la fiesta" es tan razonable como "después de terminada la fiesta"). Parecería tratarse de un contraejemplo.

Pero incluso estos casos no se confunden con las construcciones AUC laxas. Basta con sustituir el participio para que la regla se cumpla. El verbo $\varphi \alpha i ́ v o \mu \alpha \iota$ es aquí análogo a "llegar a ser". Si el verbo fuera otro, el participio no sería eliminable. Lo prueban otras frases preposicionales rigurosamente análogas, regidas por $\alpha \mu \alpha$, 
donde el participio no puede ser eliminado, verbigracia, $\alpha \mu \alpha \delta^{\prime} \eta \dot{\varepsilon} \lambda \hat{i} \omega \kappa \alpha \tau \alpha \delta v_{1} v \tau \iota$ (Hom. Il. 1. 492; Od. 16. 366), "con el sol poniéndose" o "(simultáneamente) a la

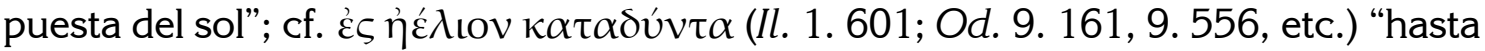

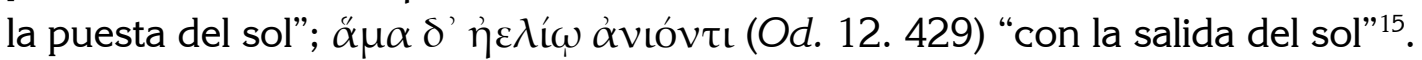

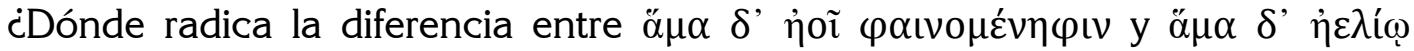
$\kappa \alpha \tau \alpha \delta u ́ v \tau$ ? No en el tipo de construcción, sino en el tipo de verbo. Aquí, una aclaración que corrige parcialmente la regla de necesidad del participio: en la construcción AUC, los participios son obligatorios salvo cuando significan "ser", "llegar a ser" o algo análogo como, en nuestro ejemplo, "aparecer" o "comenzar"16.

El participio no solo es imprescindible semánticamente; nunca funciona como una suerte de aposición. Si es posible separarlo hipotéticamente con comas o incluirlo entre paréntesis, no se tratará de un participio AUC. En $\tau \tilde{\omega}$ oít $\omega$

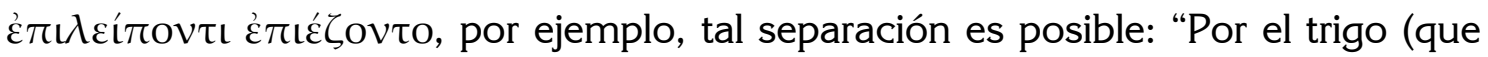

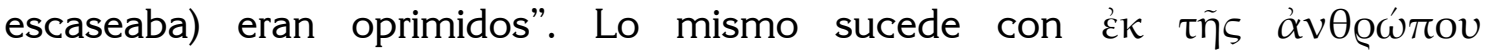
$\beta \alpha \sigma \alpha v \iota \sigma \theta \varepsilon i ́ \sigma \eta \varsigma$ (Lys. 4. 10. 3); puede entenderse que obtuvieron la información "a partir de la persona (que había sido torturada)". En cambio, es imposible traducir غ̇ $\varsigma$

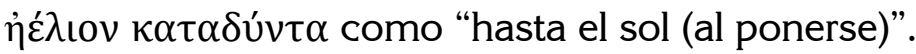

Desde luego, si se eliminan los participios de las construcciones AUC laxas se deja de lado cierta información. Esta información puede considerarse semánticamente central. Pero esto mismo puede decirse de muchos simples adjetivos predicativos. La centralidad semántica debe ser considerada en otro estadio del análisis.

6. El participio modifica al núcleo de un término preposicional. Cuando no lo hace,

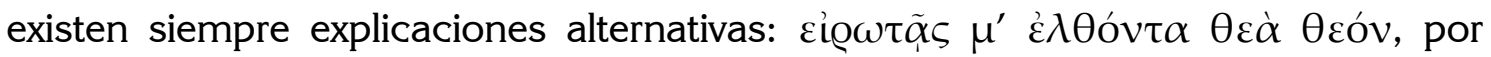
ejemplo, tiene un participio típico de discurso indirecto; no tiene por qué

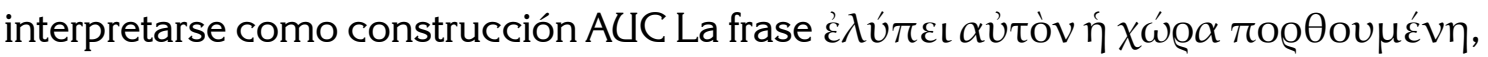
por su parte, puede entenderse como "lo apenaba la región (que estaba destruida)", no necesariamente "se apenaba por la destrucción de la región"; no solo eso: el participio podría perfectamente eliminarse.

7. Las últimas dos reglas pueden tratarse conjuntamente: el participio es predicativo, no atributivo y, ligado a esto, tiene una posición relativamente fija.

En efecto, y a diferencia de lo que ocurre en latín (cf. ante conditam condendamue urbem en el prefacio de Livio), el participio AUC en sentido estricto nunca se encuentra en posición atributiva. Los contraejemplos aparentes en, verbigracia, la sintaxis de $\mathrm{STAHL}^{17}$, constituyen casos de construcciones AUC en sentido laxo o, incluso, falsas construcciones AUC: ninguno de dichos participios es exigido por la construcción (cf. supra, § 5); todos pueden eliminarse sin que la oración se torne agramatical ni se vea tergiversada.

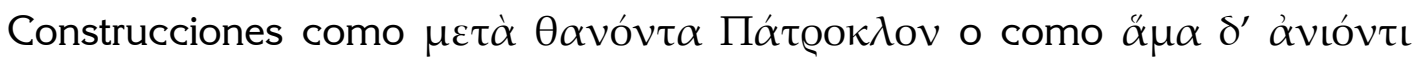

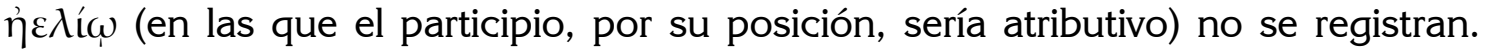
Recordemos que el orden de las construcciones también es relativamente rígido en 
castellano, si bien la regla no es extensible a todos los casos. A título de ejemplo, "Por localidades agotadas" no puede reescribirse en "Por agotadas localidades".

8. JONES presenta un aparente contraejemplo de estas tres últimas reglas: se trataría de una construcción AUC cuyo participio sería atributivo, no estaría en un complemento preposicional y (agregamos nosotros) no tendría una posición

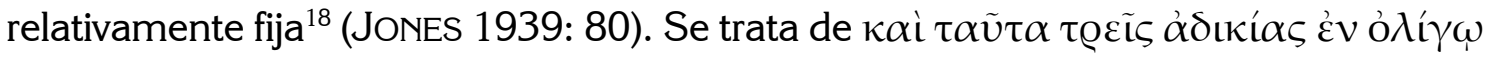

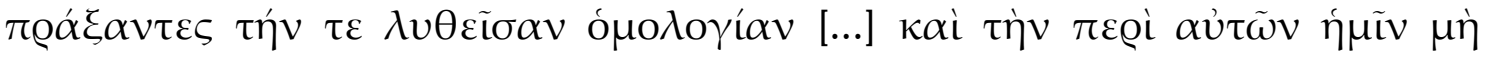

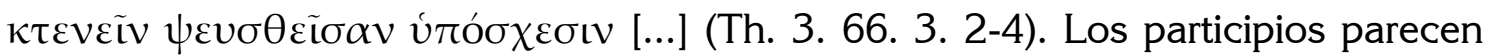
obligatorios; no se los puede eliminar mentalmente. "Cometieron injusticias ... el pacto y la promesa" provee un sentido inaceptable.

Sin embargo, si se observa con atención, puede apreciarse que la supuesta necesidad de los participios se debe a la coloración que otorgan a los sustantivos, quitándoles el carácter paradójico que de otro modo tendrían. Una traducción puede ejemplificar esta interpretación: "cometieron dos injusticias, el pacto (que violaron) y la promesa (que no respetaron)". Ni el pacto ni la promesa son, en sí, injusticias; lo son solo por el participio que los modifica. Esto puede generar la ilusión de que el participio es obligatorio sintácticamente. Pero la relación entre sustantivo y participio (atributivo) es la habitual, pese a su carácter recherché. También en determinadas ocasiones un adjetivo puede ser necesario semánticamente y por ende parecer obligatorio desde el punto de vista de la

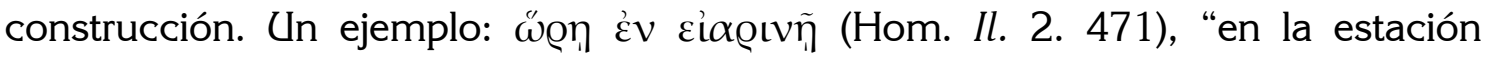
primaveral". El participio del pasaje de Tucídides es necesario como ese adjetivo, no como un participio AUC; ambos, por lo demás, tienen la peculiaridad sintáctica de que puede transformarse en atributivo sin problemas, y eso los diferencia de los participios de las construcciones AUC en sentido estricto. Que por motivos literarios convenga traducir el pasaje de Tucídides como "la violación del pacto" y "la ruptura de la promesa" no obsta a la explicación recién propuesta ${ }^{19}$.

Hemos visto que, cuando la construcción es AUC, el participio no puede ser eliminado ( $(5)$. Esto no significa que todos los participios obligatorios correspondan a una estructura AUC en sentido estricto. En el ejemplo de Tucídides alcanza con sustituir los sustantivos y sus atributos para notar que la peculiaridad reside en ellos

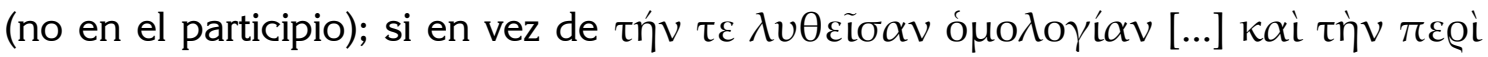

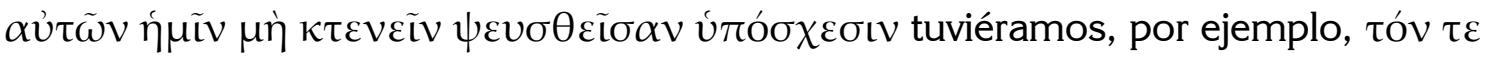

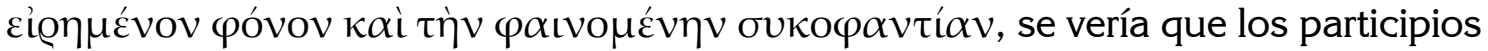
pueden eliminarse; la especificidad no radica en la construcción de participio, sino en la relación semántica entre atributo y sustantivo. Ciertamente, se podría traducir "la mención del delito y la aparición de la delación"; pero no se trataría más que de una construcción AUC en sentido laxo.

Un excursus. Tras releer los poemas homéricos en busca de participios AUC, creemos poder afirmar que, en Odisea, la construcción AUC estricta se reserva a fenómenos astronómicos (la puesta del sol o la aparición de la aurora) en estructuras 
formulaicas y fosilizadas. La única excepción que he encontrado aparece en Hom.

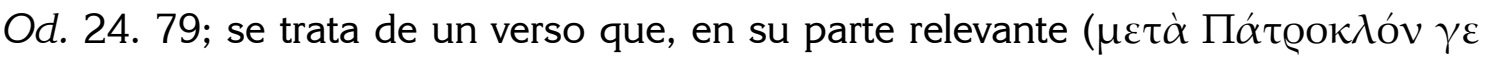
$\theta \alpha$ vóvt $\alpha$ ), es idéntico a Il. 24. 575 y posiblemente deba explicarse como un préstamo $^{20}$.

En la Ilíada, el tipo de construcción no ligada a fenómenos astronómicos puede

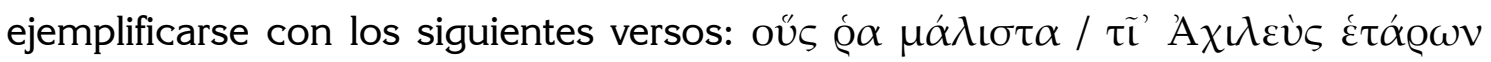

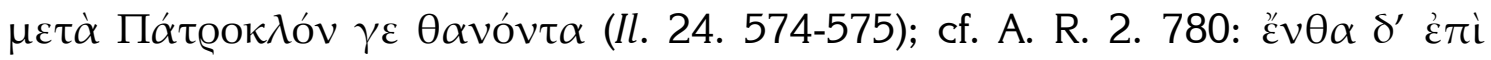

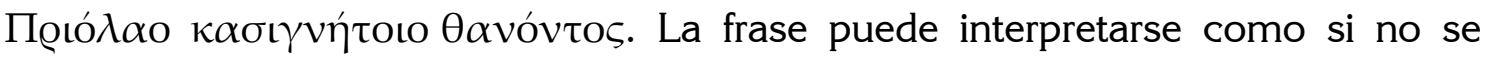
tratara de una construcción AUC: "A quienes de sus compañeros honraba sobre todo Aquiles, después de Patroclo, que había muerto". Pero esta traducción instaura una imprecisión ausente en Homero. "Después de Patroclo, que había muerto", da a entender que Aquiles seguía honrando en primer término a Patroclo. Si esta interpretación fuera cierta, el participio, que solo agregaría una característica del sustantivo en cuestión y sería explicativo, podría eliminarse mentalmente sin incurrirse en una tergiversación grave. Pero este no es el caso. El sentido es que Aquiles honra de preferencia a ellos después de la muerte de Patroclo, no como segundos en consideración después de Patroclo; se hallan en planos diferentes. Una traducción más acertada, por ende, sería: "A quienes de sus compañeros honraba sobre todo Aquiles, después de muerto Patroclo", "una vez muerto Patroclo", "después de la muerte de Patroclo" o "después de que Patroclo muriese". Este ejemplo muestra la importancia semántica y funcional de definir adecuadamente el participio de la construcción AUC, diferenciándolo de un participio predicativo cualquiera. No se trata de un mero problema de traducción, sino de la relación del participio con el resto de la frase. Un ejemplo aparentemente idéntico es, en

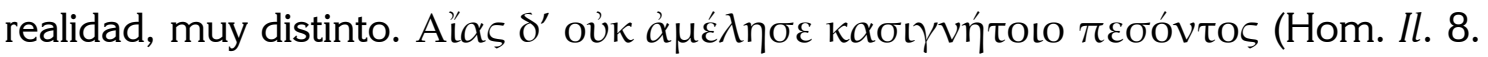
330) no es una construcción AUC en sentido estricto; puede entenderse "no descuidó a su hermano (que había caído)"; el participio mismo no es imprescindible para la significación.

\section{Bibliografía primaria}

CAREY, C. (2007). Lysiae Orationes cum fragmentis. Oxonii.

FRÄNKEL, H. (1961) Apollonii Rhodii Argonautica. Oxonii.

MARCHANT, E.C. (1921) Xenophontis Opera omnia. Oxonii.

OGILVIE, R.M. (1974) Titi Livi Ab urbe condita. Vol. 1, Libros I-V. Oxonii.

StUART JONES, H. - POWELl, J.E. (1942) Thucydidis Historiae. 2 vols. Oxonii.

WeSt, M. (1998-2000) Homeri Ilias. 2 vols. Monachi et Lipsiae.

West, M. (2017) Homerus. Odyssea. Berlin - Boston.

WiLson, N.G. (2015) Herodoti Historiae. 2 vols. Oxonii. 


\section{Bibliografía secundaria}

AlLEN, J.H. \& GREenOugh, J.B. (1903). New Latin Grammar. Boston.

ChANTRAine, P. (1953). Grammaire homérique (Vol. 2). Paris.

Classen, J. (1867). Beobachtungen über den homerischen Sprachgebrauch. Frankfurt a.M.

GILDERSLEEVE, B.L. (1892). Brief Note. American Journal of Philology 13, 257-260.

HAHN, E.A. (1928). The ab urbe condita type of expression in Greek and English. Classical Journal 23(4), 266-274.

JONES, F.P. (1939). The Ab Urbe Condita Construction in Greek: A Study in the Classification of the Participle. Language 15(1), 5-96.

SCHWYZER, E. (1950). Griechische Grammatik (Vol. 2). München.

SMYTH, H.W. (1956). Greek Grammar. Cambridge.

STAHL, J.M. (1907). Kritisch-historische Syntax des griechischen Verbums der klassischen Zeit. Heidelberg.

STEVENS, W.A. (1872). On the Substantive Use of the Greek Participle. Transactions of the American Philological Association, 3, 45-55.

Notas

${ }^{1}$ SMYTH (1956: 456, § 2053): "A participle and its substantive often correspond to a verbal noun with the genitive or to an articular infinitive. Cp. post urbem conditam and Milton's 'Since created man". La definición en una gramática latina estándar: "A noun and a passive participle are often so united that the participle and not the noun contains the main idea", ALLEN \& GREENOUGH (1903: 313, § 497).

2 SCHWYZER (1950: 404).

${ }^{3}$ Cf. JONES (1939: 7-8); se refiere a GILDERSLEEVE (1892: 258). Gildersleeve pone de relieve también la frecuencia de la construcción en Homero.

${ }^{4}$ Hay construcciones estrictamente análogas, e.g. Hom. Il. 11. 685; Od. 4. 407, 6. 31, etc. En lo que sigue considero $\ddot{\alpha} \mu \alpha$, en los casos en que acompaña un dativo, simplemente como preposición, sin detenerme en si es propia o impropia. Salvo indicación en contrario, todas las traducciones me pertenecen.

${ }^{5}$ STEVENS (1872: 49) opina, al analizar conjuntamente este ejemplo tucidídeo, el de Hom. Il. 1. 601 (que veremos infra) y otros de prosistas áticos y jónicos, lo siguiente: "The participle may be used substantively, depending either with its subject, or alone, on a preposition or an adverb". Véase también HAHN (1928).

${ }^{6}$ Debo esta última oración al atento referato de uno de los evaluadores anónimos.

${ }^{7}$ Cf. JONES (1939: 15). 
8 JONES (1939: 13). Para ejemplos en lenguas modernas, cf. ibid., p. 6, n. 2. También en castellano hay construcciones AUC, como "después de abierta la puerta", que puede reescribirse como "después de la apertura de la puerta", o "después de finalizada la función", reescribible como "tras la finalización de la función". Formulaciones algo artificiosas son perfectamente comprensibles y probablemente idiomáticas: "antes de fundada la ciudad" puede traducir una frase de Livio (ante conditam... urbem).

9 SCHWYZER (1950: 404).

10 SCHWYZER 1950: 393, § 4

11 SMYTH (1956) señala que esta construcción, al igual que otras análogas, puede

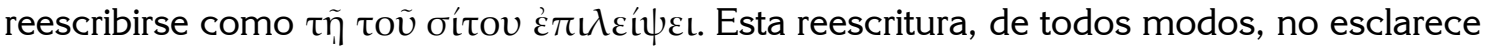
las características específicas de las construcciones.

${ }^{12}$ Estos dos ejemplos aparecen ya en GILDERSLEEVE (1892: 258-259), junto con varios que no constituyen construcciones a.u.c. en sentido estricto y uno con resonancias homéricas

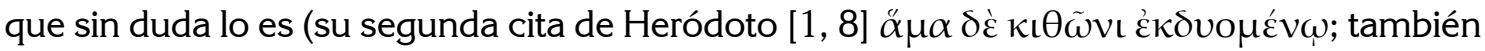
son construcciones a.u.c. en sentido estricto sus citas de Antifonte, Platón y Jenofonte).

${ }^{13}$ Quizá podría agregarse un quinto rasgo: el participio es de un verbo intransitivo o está en voz pasiva.

${ }^{14}$ SMYTH (1956: 455, § 2046): "Supplementary: as a supplement to a verbal predicate, which, without such a supplement, would be incomplete". Para las excepciones, que son análogas a los de las construcciones AUC (el participio puede ser sobreentendido en ciertos casos, particularmente con el verbo eìuí), cf. infra, n. 17.

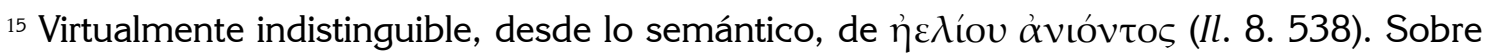
las diferencias de aspecto entre los participios infectivos y confectivos (aoristos) de estas construcciones, cf. las astutas notas de CLASSEN (1867: 59).

${ }_{16}$ Algo semejante sucede, desde luego, con otras construcciones clásicas que exigen

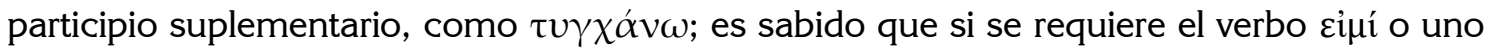

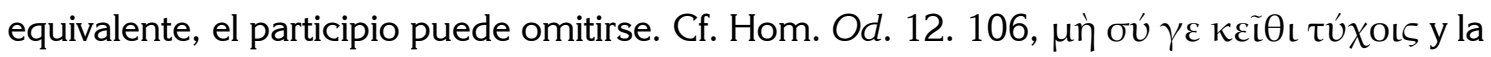
explicación de CHANTRAINE (1953: 326, "ne va pas être là").

17 STAHL (1907: 697-698).

18 JONES (1939: 80).

${ }^{19}$ Los otros dos ejemplos en los que Jones pretende identificar un participio AUC en posición atributiva pueden explicarse del mismo modo.

20 Hay una variatio en Hom. Od. 24. 77: el participio confectivo $\theta \alpha v \omega ́ v$ se aplica nuevamente a Patroclo pero, aquí, se trata de un simple participio predicativo. 